\title{
Vertical monolithic integration of wide- and narrow-bandgap semiconductor nanostructures on graphene films
}

\author{
Youngbin Tchoe ${ }^{1}$, Janghyun Jo², HoSung Kim³ ${ }^{3}$ Heehun Kim', Hyeonjun Baek', Keundong Lee', Dongha Yoo', \\ Won Jun Choi ${ }^{4}$ Miyoung Kim² and Gyu-Chul Yi ${ }^{1}$
}

\begin{abstract}
We report monolithic integration of indium arsenide ( $\mathrm{InAs}$ ) nanorods and zinc oxide $(\mathrm{ZnO})$ nanotubes using a multilayer graphene film as a suspended substrate, and the fabrication of dual-wavelength photodetectors with the hybrid configuration of these materials. For the hybrid nanostructures, $\mathrm{ZnO}$ nanotubes and InAs nanorods were grown vertically on the top and bottom surfaces of the graphene films by metal-organic vapor-phase epitaxy and molecular beam epitaxy, respectively. The structural, optical, and electrical characteristics of the hybrid nanostructures were investigated using transmission electron microscopy, spectral photoresponse analysis, and current-voltage measurements. Furthermore, the hybrid nanostructures were used to fabricate dual-wavelength photodetectors sensitive to both ultraviolet and mid-infrared wavelengths.
\end{abstract}

\section{Introduction}

The monolithic integration of wide-bandgap and narrow-bandgap semiconductors is of great interest, since it broadens the spectral absorption range of lightharvesting devices and photodetectors ${ }^{1-4}$. Additionally, the unique properties of each material in the integrated system can be synergistically combined to create multifunctional devices. Along this line of research, many stateof-the-art tandem solar cells and photodetectors have been produced under various combinations of $\mathrm{Si}, \mathrm{II}-\mathrm{VI}$, and III-V semiconductors ${ }^{5-7}$, quantum $\operatorname{dots}^{8}$, quantum wells ${ }^{9}$, two-dimensional materials ${ }^{10}$, and, more recently, perovskite $^{11}$ and organic materials ${ }^{12}$. However, the integration

\footnotetext{
Correspondence: Gyu-Chul Yi (gcyi@snu.ac.kr)

'Department of Physics and Astronomy, Institute of Applied Physics, and Research Institute of Advanced Materials, Seoul National University, Seoul, Korea

${ }^{2}$ Department of Material Science and Engineering and Research Institute of Advanced Materials, Seoul National University, Seoul, Korea
}

Full list of author information is available at the end of the article of wide-bandgap and narrow-bandgap semiconductors via the direct growth approach has presented a significant challenge, since heteroepitaxy works well only between two semiconductor layers with small differences between their lattice parameters and thermal expansion coefficients ${ }^{13,14}$. Although wafer bonding can be used to glue two different semiconductors together ${ }^{15-17}$, the highquality semiconductor heterostructures with a defect-free and clean interface required for many sophisticated device applications can generally only be prepared by direct growth based on heteroepitaxy ${ }^{18,19}$. In this work, to demonstrate monolithic integration of narrow-bandgap and wide-bandgap semiconductors, we report the growth of indium arsenide (InAs) nanorods and zinc oxide ( $\mathrm{ZnO})$ nanotubes on the top and bottom surfaces of a suspended graphene film. Furthermore, the hybrid nanostructures were used to fabricate dual-wavelength photodetectors sensitive to both ultraviolet and mid-infrared wavelengths. Our technique for integrating wide-bandgap and narrowbandgap semiconductors is expected to greatly increase the versatility and power of these building blocks for applications in optoelectronics and photonics.

\section{(c) The Author(s) 2021}

(c) (i) Open Access This article is licensed under a Creative Commons Attribution 4.0 International License, which permits use, sharing, adaptation, distribution and reproduction cc) in any medium or format, as long as you give appropriate credit to the original author(s) and the source, provide a link to the Creative Commons license, and indicate if changes were made. The images or other third party material in this article are included in the article's Creative Commons license, unless indicated otherwise in a credit line to the material. If material is not included in the article's Creative Commons license and your intended use is not permitted by statutory regulation or exceeds the permitted use, you will need to obtain permission directly from the copyright holder. To view a copy of this license, visit http://creativecommons.org/licenses/by/4.0/. 


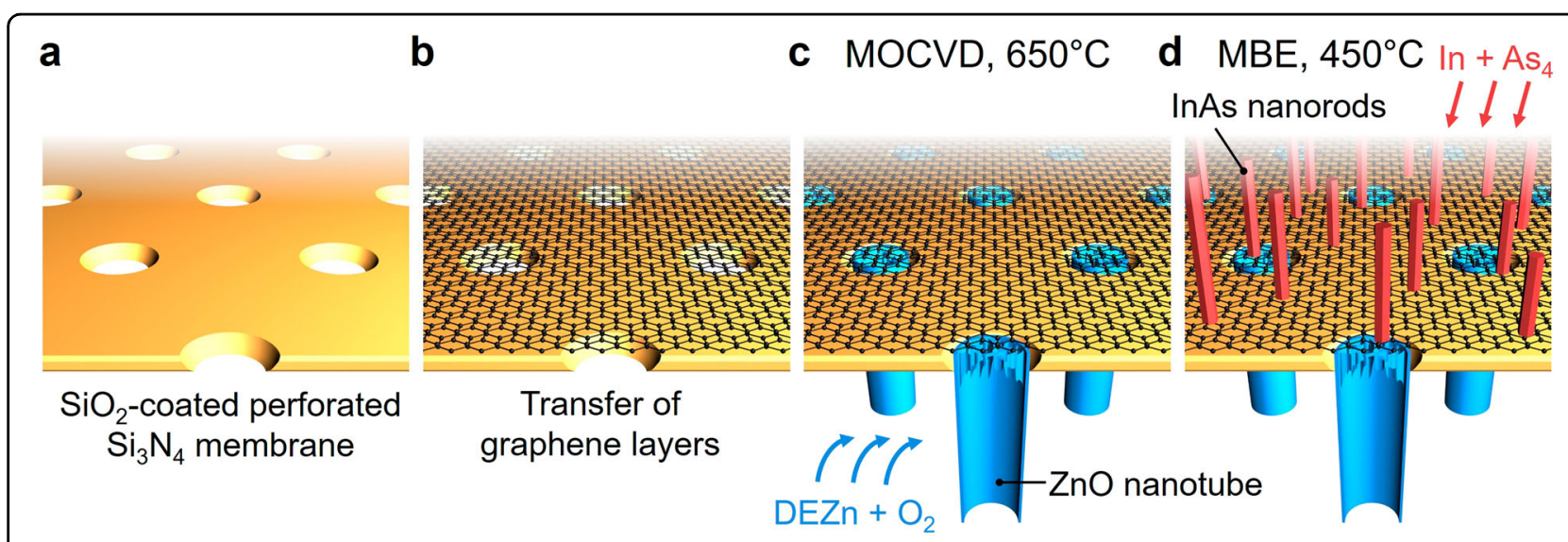

Fig. 1 Schematic diagram of the fabrication processes of indium arsenide (InAs) nanorod/graphene layers/zinc oxide ( $\mathrm{ZnO})$ nanotube hybrid nanostructures. a Transfer of chemical vapor-deposited graphene layers onto a hole-patterned $\mathrm{SiO}_{2} / \mathrm{Si}_{3} \mathrm{~N}_{4}$ membrane. $\mathbf{b}$ Chemical vapordeposited graphene layers transferred onto a $\mathrm{SiO}_{2} / \mathrm{Si}_{3} \mathrm{~N}_{4}$ membrane. $\mathbf{c} \mathrm{ZnO}$ nanorod array growth on the backside using selective-area metal-organic vapor-phase epitaxy (SA-MOVPE). d Catalyst-free molecular beam epitaxy (MBE) growth of InAs nanorods on the front side. e Cross-sectional schematic structures of the InAs nanorod/graphene layers/ZnO nanorod hybrid nanostructure.

\section{Results and discussion}

The basic approach for growing InAs nanorods and $\mathrm{ZnO}$ nanotubes on the top and bottom surfaces of suspended graphene layers and the detailed fabrication steps are schematically illustrated in Fig. 1. Suspended multilayer graphene (MLG, $n=10$ ) with full coverage was synthesized by chemical vapor deposition (CVD) and transferred onto perforated silicon dioxide $\left(\mathrm{SiO}_{2}\right)$-coated silicon nitride $\left(\mathrm{Si}_{3} \mathrm{~N}_{4}\right)$ membranes with 300 -nm-diameter hole arrays (see Fig. 1a, b). The $\mathrm{SiO}_{2} / \mathrm{Si}_{3} \mathrm{~N}_{4}$ membrane with small hole openings ensured mechanically stable and flat preparation of suspended graphene layers over a relatively large area ${ }^{20}$, while minimizing the potential for mechanical damage such as cracks or tears. The suspended graphene substrate was loaded upside down into a metal-organic chemical vapor deposition (MOCVD) system for growth of $\mathrm{ZnO}$ nanotubes. In this configuration, the perforated $\mathrm{SiO}_{2} / \mathrm{Si}_{3} \mathrm{~N}_{4}$ membrane masked the graphene layers from the reactants, and vertical $\mathrm{ZnO}$ nanotubes were grown at $650^{\circ} \mathrm{C}$ by selective-area metalorganic vapor-phase epitaxy (SA-MOVPE), as shown in Fig. 1c. This selective-area growth is attributed to the preferred nucleation and crystal growth of $\mathrm{ZnO}$ at step edges on graphene films ${ }^{21}$ and weaker interaction of $\mathrm{Zn}$ adatoms with the amorphous $\mathrm{SiO}_{2}$ surface, which leads to higher desorption rate and diffusion of $\mathrm{Zn}$ adatoms on $\mathrm{SiO}_{2}$ compared to those on the graphene film ${ }^{22,23}$. Figure 1c also schematically illustrates the existence of $\mathrm{ZnO}$ nanowall structures inside the nanotubes, which are usually observed for selective-area-grown $\mathrm{ZnO}$ nanotubes with diameters of a few hundred nanometers ${ }^{22}$. After the $\mathrm{ZnO}$ nanotubes were grown on one side of the graphene layers, the substrate was inverted to expose the other side and loaded into a molecular beam epitaxy (MBE) system.
Since the $\mathrm{ZnO}$ nanotubes were grown inside the $300 \mu \mathrm{m}$ recessed cavities in the Si substrate, the sample loading and unloading process did not physically damage the $\mathrm{ZnO}$ nanotubes. In the MBE system, vertical InAs nanorods were grown on the opposite side of the graphene layers using a catalyst-free growth method, enabling monolithic integration of $\mathrm{ZnO}$ nanotubes and InAs nanorods on the suspended graphene film, as shown in Fig. 1d.

The morphologies of the InAs nanorods and $\mathrm{ZnO}$ nanotubes grown on each surface of the graphene layers were investigated using scanning electron microscopy (SEM). Figure 2a shows an SEM image of free-standing $\mathrm{ZnO} / \mathrm{MLG} / \mathrm{InAs}$ hybrid nanostructures, revealing the formation of $\mathrm{ZnO}$ nanotubes and InAs nanorods on the top and bottom surfaces of the graphene layers. Both the $\mathrm{ZnO}$ nanotubes and InAs nanorods were vertically well aligned on their respective surfaces on the suspended graphene layers. As shown in Fig. 2b, it is evident that $\mathrm{ZnO}$ nanotubes were selectively grown only on the graphene layers through the $\mathrm{SiO}_{2} / \mathrm{Si}_{3} \mathrm{~N}_{4}$ membrane growth mask. The diameter, spacing, and length of the $\mathrm{ZnO}$ nanotubes were $0.3,2$, and $3 \mu \mathrm{m}$, respectively. The dimensions of the $\mathrm{ZnO}$ nanotubes could easily be controlled by changing the diameter and spacing of the hole arrays and the growth time of $\mathrm{ZnO}$. On the other side of the graphene film, as shown in Fig. 2c, high-density vertical InAs nanorods with an average length of $3 \mu \mathrm{m}$, a diameter of $50 \mathrm{~nm}$, and a density of $5 \mu \mathrm{m}^{-1}$ were formed. SEM images showed that the density of the InAs nanorods was uniform across the graphene layers and was not affected by the presence of the $\mathrm{ZnO}$ nanotubes grown on the other side. This observation suggests that the MLG $(n=10)$ screened the interaction ${ }^{19}$ between the two different semiconductors, and that the nucleation of the 


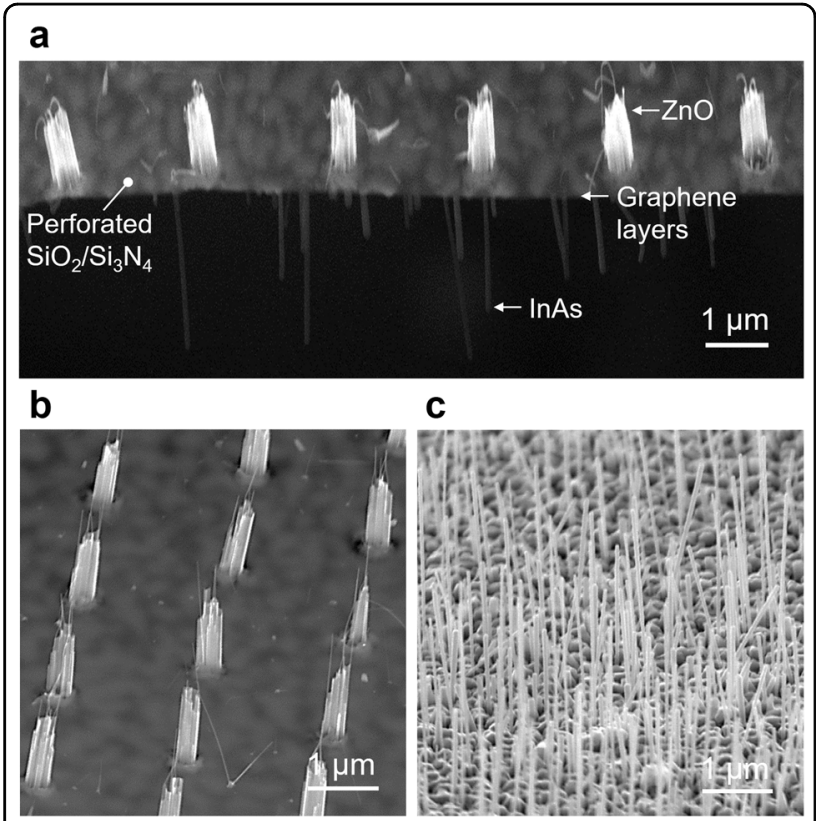

Fig. 2 Morphology of the InAs nanorod/graphene layers/ZnO nanorod hybrid nanostructure. Tilted field-emission scanning electron microscopy (FE-SEM) images of $\mathbf{a}$ the cross-section, $\mathbf{b}$ the $\mathrm{ZnO}$ nanostructure array on the front side, and $\mathbf{c}$ the InAs nanorods on the backside.

InAs nanorods was not affected by the $\mathrm{ZnO}$ nanotubes grown on the other side.

In addition to the $\mathrm{ZnO} / \mathrm{MLG} / \mathrm{InAs}$ heterostructures, we also demonstrated gallium nitride (GaN) microrod/MLG/ InAs heterostructures using the same integration strategy (see Fig. S1). The GaN microrods grown on graphene layers ${ }^{24}$ were transferred onto a Si substrate with $300 \times$ $300 \mu \mathrm{m}^{2}$ square window openings; the sample was loaded upside down into the MBE system, and InAs nanorods were grown on the back side of the exposed graphene layers. The SEM image in Fig. S1 reveals that InAs nanorods and $\mathrm{GaN}$ microrods were vertically integrated through the thin graphene layers. In this work, the integration of $\mathrm{ZnO}$ nanotubes or $\mathrm{GaN}$ microrods with InAs nanorods is presented as a representative example, since these materials are difficult to integrate using conventional growth approaches due to the very large differences in their physical characteristics. Although only two combinations were demonstrated in this work, we strongly believe that a greater variety of materials can be monolithically integrated using the same approach.

The microstructural characteristics of the InAs/MLG/ $\mathrm{ZnO}$ hybrid nanostructures were investigated using transmission electron microscopy (TEM). The bright-field (BF) cross-sectional TEM in Fig. 3a clearly shows an integrated structure composed of $\mathrm{ZnO}$ nanotubes with nanowalls vertically grown on top of the MLG through the $\mathrm{SiO}_{2} / \mathrm{Si}_{3} \mathrm{~N}_{4}$ membrane, and InAs nanorods and nanoislands grown on the opposite side of the MLG. The atomic arrangement and interfacial layers between the hybrid InAs/MLG/ZnO layers were also examined using high-resolution TEM (HR-TEM), as shown in Fig. 3b. The HR-TEM image clearly indicates that the InAs nanorods and $\mathrm{ZnO}$ nanotubes were grown as single crystals on each side of the MLG without any interfacial layers or gaps. The lattice spacings between adjacent planes were estimated to be $0.26,0.34$, and $0.35 \mathrm{~nm}$, corresponding to the $d$-spacings of $\mathrm{ZnO}(0002)$, graphene layers(0002), and InAs(111) planes ${ }^{22,25}$. Additionally, compositional analysis conducted using scanning TEM (STEM) equipped with an energy dispersive X-ray spectroscopy system (Fig. S2) showed that there was no interdiffusion through the interface layers, and that the graphene films effectively prevented In and $\mathrm{As}_{4}$ fluxes from passing through to the other side of the graphene layers during growth.

The crystallinity of the heterostructure was further investigated by analyzing diffraction patterns (DPs) with a selective aperture size of $150 \mathrm{~nm}$. Figure 3c-e show the DPs from the $\mathrm{ZnO}$ nanotubes, InAs nanorods, and $\mathrm{ZnO} /$ MLG/InAs interfaces, respectively. The DPs in Fig. 3c, d were measured along the zone axis near the [2 $\overline{1} 10]$ direction of $\mathrm{ZnO}$ and $[11 \overline{2} 0]_{\mathrm{WZ}}$ and $[1 \overline{1} 0]_{\mathrm{ZB}}$ directions of InAs, respectively. The patterns revealed that $\mathrm{ZnO}$ nanotubes with a wurtzite single crystal structure and a $c$ axis orientation grew normal to the graphene layers. On the other hand, streak patterns were observed in the DP of the InAs nanorods as a result of the stacking faults typically observed in InAs nanorods ${ }^{25,26}$. The InAs nanorods exhibited $(111)_{\mathrm{ZB}}$ and $(0002)_{\mathrm{WZ}}$ orientations normal to the graphene. The DP near the $\mathrm{ZnO} / \mathrm{MLG} / \mathrm{InAs}$ interfaces showed diffraction peaks of $\mathrm{ZnO}$ and InAs that were exactly the same as those in the overlapped DPs of crystalline $\mathrm{ZnO}$ and InAs shown in Fig. 3c, d, respectively. In addition to the DP analysis, the fast Fourier transform of the HR-TEM image (see Fig. S3) revealed the alignment of the $\mathrm{C}(0002)$ peak with the $\mathrm{ZnO}(0002)$ and $\operatorname{InAs}(111)_{\mathrm{ZB}}$, $(0002)_{\mathrm{WZ}}$ peaks. This result indicates that the $\mathrm{ZnO}$ nanotubes and InAs nanorods were heteroepitaxially grown on graphene layers with an epitaxial relationship of $\operatorname{InAs}(111)_{\mathrm{ZB}},(0002)_{\mathrm{WZ}}\|\mathrm{MLG}(0002)\| \mathrm{ZnO}(0002)$.

The in-plane epitaxial relationships among the InAs nanorods, MLG, and $\mathrm{ZnO}$ nanotubes were investigated by plan-view TEM. For these experiments, we prepared InAs/MLG/ZnO hybrid heterostructures on graphene layers with nanoscale $\mathrm{ZnO}$ and InAs nuclei, which are small enough for electron beam transmission. These nuclei were grown on graphene layers for a very short time such that their average sizes were controlled to be less than $50 \mathrm{~nm}$, and the suspended graphene layers were electron beam transparent. Figure $3 f$ shows a plan-view HR-TEM image of the hybrid heterostructure near the point of intersection of InAs, MLG, and $\mathrm{ZnO}$ single 

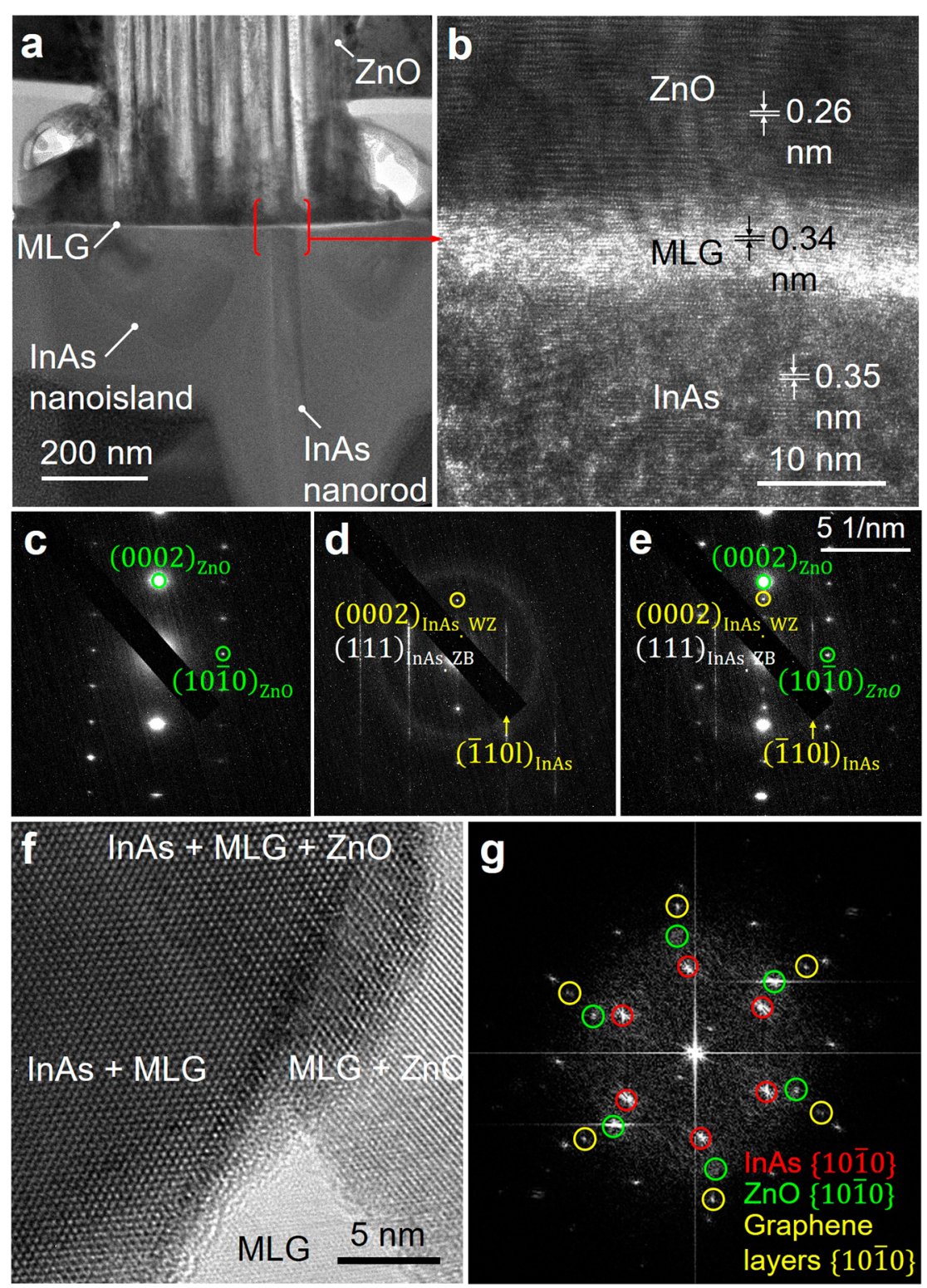

Fig. 3 Cross-sectional transmission electron microscopy (TEM) analysis of the hybrid heterostructure. a Bright-field and $\mathbf{b}$ high-resolution (HR)-TEM images of the hybrid heterostructures near the ZnO nanorod/multilayer graphene (MLG)/InAs nanorod interfaces. Diffraction patterns acquired using a selective aperture size of $150 \mathrm{~nm}$ near the $\mathbf{c} \mathrm{ZnO}$ nanorod, $\mathbf{d}$ InAs nanorod, and $\mathbf{e} \mathrm{ZnO} / \mathrm{MLG} / \mathrm{InAs}$ interfaces. Plan-view TEM structural analysis of the heterostructure. $\mathbf{f}$ Plan-view HR-TEM image showing overlap of InAs and ZnO nuclei. $\mathbf{g}$ Corresponding fast Fourier transform of $\mathbf{f}$.

crystals; the hexagonal lattice structures of InAs, $\mathrm{ZnO}$, and MLG are clearly visible in this HR-TEM image. On the upper side of the HR-TEM image, all three crystals, InAs/MLG/ZnO, overlapped, while on the left or right side of the image, two crystals (either InAs/MLG or MLG/ $\mathrm{ZnO})$ overlapped. There were no overlapping semiconductor crystals on the lower side of the HR-TEM image where only the periodic lattice structure of MLG is observed. We analyzed the fast Fourier transform (FFT) (see Fig. 3g) of the HR-TEM image to determine the inplane orientations of InAs, $\mathrm{ZnO}$, and MLG and confirmed that both the $\mathrm{ZnO}$ and InAs nanostructures were grown heteroepitaxially on the graphene layers with an in-plane heteroepitaxial relationship of InAs (1010) || MLG $(10 \overline{1} 0) \| \mathrm{ZnO}(10 \overline{1} 0)$. These results again suggest that graphene film could be an excellent heteroepitaxial substrate to integrate many different semiconductor crystals.

A vertical photodetector was fabricated as a representative example of a device application using the InAs nanorod/MLG/ZnO nanotube hybrid heterostructures. Figure 4a schematically illustrates the device structure, where gold $(\mathrm{Au})$ electrodes, as Schottky contacts, were 

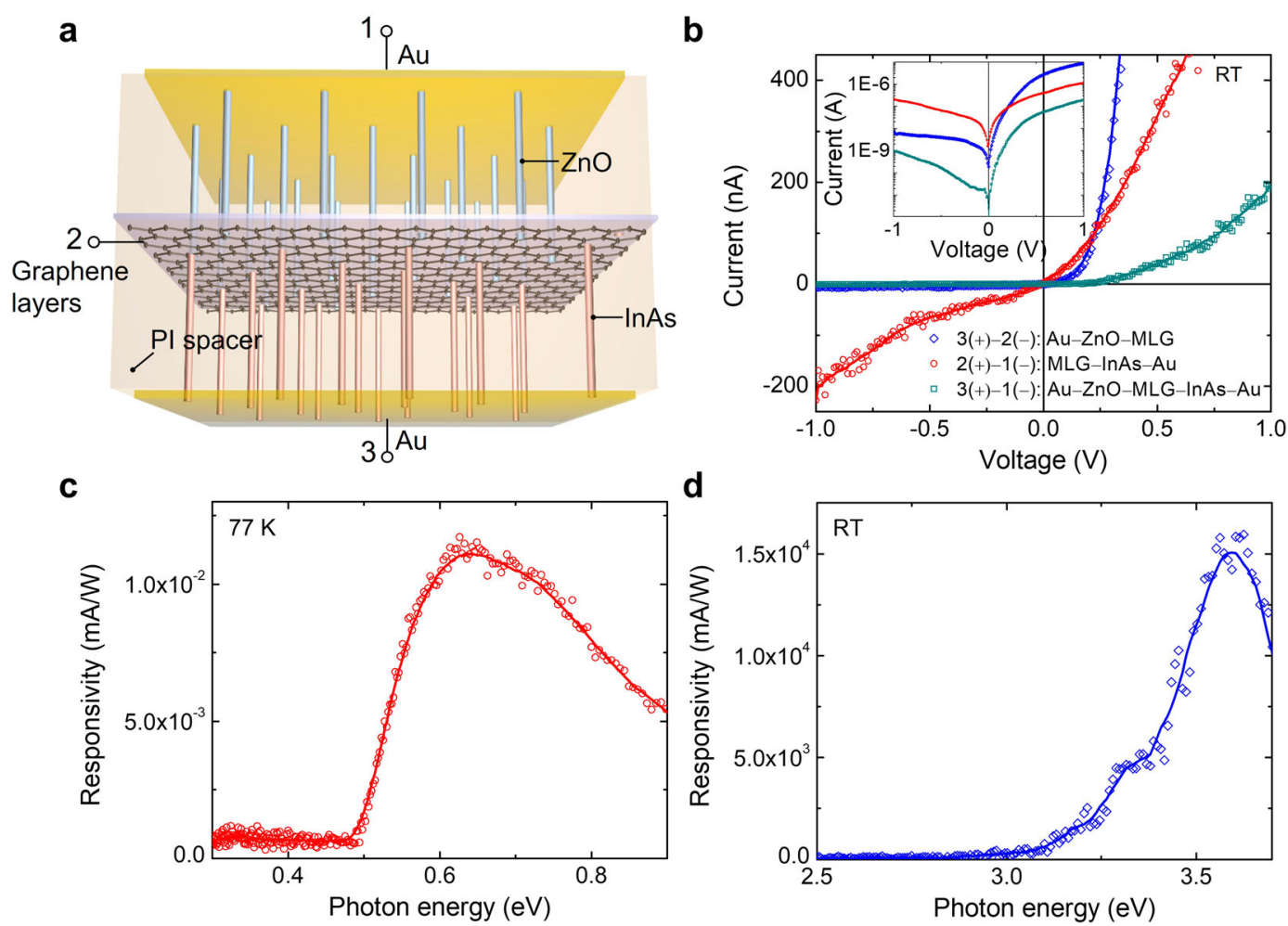

Fig. 4 Photodetector device. a Schematic diagram of the device structure, and $\mathbf{b}$ current-voltage characteristic curves measured between three electrodes at room temperature (RT). Inset figure shows the semi-log plot. c Spectral response of the gold (Au)-InAs nanorod-graphene layers measured at $77 \mathrm{~K}$ using Fourier transform-infrared (FT-IR) spectroscopy. $\mathbf{d}$ Spectral response of the Au-ZnO nanorod-graphene layers measured at RT.

formed on the tips of the InAs nanorods and $\mathrm{ZnO}$ nanotubes, and silver paste provided ohmic contact to the CVD-graphene layers. For electrical isolation between the electrodes, $2-\mu \mathrm{m}$-thick polyimide (PI) layers were formed as spacers on both the top and bottom sides of the graphene layers, and oxygen plasma etching was performed to selectively expose the tips of the InAs and $\mathrm{ZnO}$ nanorods (see Fig. S4).

The current-voltage $(I-V)$ characteristic curves between the three electrodes are shown in Fig. 4b. Schottky diode characteristics with clear rectifying behavior were observed for the $I-V$ curve measured for the $\mathrm{Au}-\mathrm{ZnO}$ nanotube-MLG device. This is expected, as $\mathrm{Au}$ and $\mathrm{ZnO}$ nanotubes are capable of forming high-quality Schottky contacts ${ }^{27}$, and $\mathrm{ZnO}$ nanotubes grown on graphene layers form ohmic contacts with the graphene layers $^{22}$. Meanwhile, the $I-V$ curve for the Au-InAs nanorod-MLG device was nonlinear, as is the curve typical for two-terminal devices with Schottky contacts on both sides; slightly higher current levels were observed at forward bias voltages. This observation agrees with previous results, in which InAs nanorods generally exhibited a higher Schottky barrier for a metal contact with a lower work function; the work functions of the $\mathrm{Au}$ and graphene layers are 5.2 and $4.5 \mathrm{eV}$, respectively ${ }^{28,29}$. The $I-V$ curves of the Au-InAs nanorod-MLG device measured at $10 \mathrm{~K}$ (Fig. S5) showed clear rectifying behavior with suppression of a large thermionic current typical for narrowbandgap semiconductor-based devices. The $I-V$ characteristic curve for the $\mathrm{Au}-\mathrm{ZnO}-\mathrm{MLG}$ - InAs-Au device appeared as if the above two devices were connected in series; the overall resistance of each system was additive, and clear rectifying behavior was observed based on the dominance of the $\mathrm{Au}-\mathrm{ZnO}$ Schottky contact.

We measured the spectral responsivity of the Schottky photodiodes to determine the spectral absorption range of the hybrid photodiode. As shown in Fig. 4c, the spectral response of the InAs nanorod-side device (AuInAs-MLG) generated a photocurrent above a photon energy of $0.5 \mathrm{eV}$, and the peak responsivity was observed near $0.6 \mathrm{eV}$, similar to previously reported values ${ }^{30}$. Meanwhile, the spectral response of the $\mathrm{ZnO}$ nanotubeside Schottky photodiode ( $\mathrm{Au}-\mathrm{ZnO}-\mathrm{MLG}$ ) showed that a photocurrent was observed above a photon energy of $3.1 \mathrm{eV}$, and the responsivity peaked near $3.6 \mathrm{eV}$ (Fig. 4d). The cutoff energy was close to $3.1 \mathrm{eV}$, which is similar to previously reported values ${ }^{31}$, and indicates the selective spectral sensitivity of $\mathrm{ZnO}$ nanorod devices to UV light. Although we characterized the mid-IR photodetector characteristics of the InAs nanorod-side device with the 
InAs side facing up because of the sampling loading protocol of the FT-IR system, we expect the device to work equivalently well with the $\mathrm{ZnO}$ side facing up due to the mid-IR transparency of both $\mathrm{ZnO}^{32}$ and graphene layers ${ }^{33}$. These results strongly suggest that the device made with these hybrid heterostructures, composed of wide-bandgap and narrow-bandgap semiconductor nanorods integrated on graphene layers, can monolithically combine each material's unique optoelectronic properties, capturing photons over a wide spectral range and generating photocurrents. Such characteristics are highly desirable for broadband light-harvesting device applications.

In conclusion, we demonstrated monolithic heteroepitaxial growth of single-crystalline wide-bandgap and narrow-bandgap semiconductor nanostructures on the top and bottom surfaces of graphene layers. The graphene layers provided vertical alignment and a heteroepitaxial relationship to both InAs nanorods and $\mathrm{ZnO}$ nanotubes. Cross-sectional and plan-view TEM analyses directly showed the heteroepitaxial relationship in the $\mathrm{ZnO}$ nanotube/graphene layers/InAs nanorod hybrid heterostructure. Moreover, dual-wavelength photodetectors were fabricated using these hybrid heterostructures, and a clear optoelectronic response to UV and mid-IR light was observed. We believe that this general approach of combining various semiconductor nanostructures can be applied to many other hybrid nanostructures with unique physical properties that can be used for advanced optoelectronic device applications, such as tandem cells or multicolor light emitters.

\section{Experimental methods}

\section{Preparation of graphene layers}

Graphene films were deposited on $\mathrm{Cu}$ foil using CVD and spin-coated with PMMA. The PMMA-supported graphene was transferred onto a hole-patterned $\mathrm{SiO}_{2} /$ $\mathrm{Si}_{3} \mathrm{~N}_{4}$ membrane, and the PMMA layer was removed by organic solvents. The hole-patterned $\mathrm{SiO}_{2} / \mathrm{Si}_{3} \mathrm{~N}_{4}$ membrane was prepared by anisotropic wet etching of a $\mathrm{Si}_{3} \mathrm{~N}_{4}$ coated $\mathrm{Si}$ wafer, hole patterning by electron beam lithography, dry etching, and plasma-enhanced CVD of a 50nm-thick $\mathrm{SiO}_{2}$ layer on the hole-patterned $\mathrm{Si}_{3} \mathrm{~N}_{4}$ membrane. Raman spectroscopy and cross-sectional TEM confirmed the successful preparation of graphene layers and their layer number $(n=10)$.

\section{Selective-area metal-organic vapor-phase epitaxy of $\mathrm{ZnO}$ nanorods on graphene layers}

$\mathrm{ZnO}$ nanotubes were selectively grown on graphene layers using catalyst-free MOVPE. Diethylzinc (DEZn) and high-purity $\mathrm{O}_{2}(>99.9999 \%)$ were used as reactants, and high-purity $\operatorname{Ar}(>99.9999 \%)$ was the carrier gas. The flow rates of DEZn and $\mathrm{O}_{2}$ were 30 and $90 \mathrm{sccm}$, respectively. During growth, Ar flowed into the quartz reactor through a DEZn bubbler held at $-15^{\circ} \mathrm{C}$. To prevent premature reaction, the $\mathrm{O}_{2}$ gas line was separated from the main gas manifold line. The reactor pressure was kept at 0.3 Torr during growth, and the temperature was $650^{\circ} \mathrm{C}$. More details on the experimental setup of the MOVPE growth system can be found elsewhere ${ }^{12}$.

\section{Molecular beam epitaxial growth of InAs nanorods on graphene layers}

Inside of a cryogenically cooled ultrahigh-vacuum growth chamber (RIBER 32P), InAs nanorods were grown at $450^{\circ} \mathrm{C}$ for $35 \mathrm{~min}$ by supplying high-purity In and uncracked arsenic $\left(\mathrm{As}_{4}\right)$ molecular beams from Knudsen cells. The beam-equivalent pressures of In and $\mathrm{As}_{4}$ were $1 \times 10^{-7}$ and $3 \times 10^{-5}$ Torr, respectively. For catalyst-free growth of InAs nanorods, we supplied $\mathrm{As}_{4}$ to the substrates for $10 \mathrm{~min}$ before supplying In to prevent In droplet formation on the graphene layers. More details of the experimental setup of the MBE growth system can be found elsewhere ${ }^{34}$.

\section{Surface morphology and microstructural characterization}

The morphology of the $\mathrm{ZnO}$ nanorod/graphene layers/ InAs nanorod hybrid heterostructure was investigated using FE-SEM (MIRA3; TESCAN and AURIGA; Carl Zeiss). For detailed microstructural analysis, the crosssection was prepared by dual-beam FIB (Quanta 3D; FEG) and analyzed using TEM (Tecnai F20; FEI). The chemical composition was analyzed by high-angle annular darkfield STEM-EDX (JEM 2100F; JEOL). The plan-view TEM specimen was prepared by growing nanoscale $\mathrm{ZnO}$ and InAs nuclei on each side of the graphene layers. $\mathrm{ZnO}$ nuclei were grown only for $2 \mathrm{~min}$, and InAs nuclei were grown for $30 \mathrm{~s}$. The crystallinity of the hybrid heterostructure was investigated by BF and HR-TEM.

\section{Vertical device fabrication}

The vertical photodetector device was fabricated using InAs nanorod/graphene layers/ $\mathrm{ZnO}$ nanorod hybrid heterostructures. Prior to metallization, PI layers were coated on both sides of the InAs/MLG/ZnO structure, and oxygen plasma was used to expose the tips of the $\mathrm{ZnO}$ and InAs nanorods through the PI layers. Gold electrodes with thicknesses of 20 and $50 \mathrm{~nm}$ were deposited on the $\mathrm{ZnO}$ and InAs nanorods, respectively. The transmittance of the 20-nm-thick Au layer at $300 \mathrm{~nm}$ was estimated to be $20 \%$, and that of the $50-\mathrm{nm}$-thick Au layer at $2500 \mathrm{~nm}$ was estimated to be $0.2 \%^{35,36}$. Silver paste was used to provide ohmic contact to the CVD-graphene layers ${ }^{37}$, which was confirmed by two-probe measurements.

\section{Photodetector characterization}

The spectral response of the $\mathrm{ZnO}$ nanorod/graphene layers photodetector was measured by recording the photocurrent as a function of wavelength for monochromatic light irradiated on the device. Tunable-wavelength 
monochromatic light was generated by a $300-\mathrm{W}$ xenon lamp and a monochromator (DW150i; Dongwoo Optron), and the spectral power of the monochromatic light was recorded by a Si photodetector (S120VC; Thorlabs). The spectral response of the InAs nanorod/graphene layers photodetector was measured at $77 \mathrm{~K}$ using FT-IR spectroscopy (VERTEX 80v; Bruker), and the photocurrent was detected by a low-noise current amplifier (Keithley 428). In the FT-IR system, Globar was used as an infrared light source, together with a $\mathrm{KBr}$ beam splitter. An InAsSb photodetector (P13894-011NA; Hamamatsu) was used to measure the spectral intensity of the light source.

\section{Acknowledgements}

This work was financially supported by the Samsung Research Funding Center of Samsung Electronics (SRFC-TA1803-02(0417-20180116)).

\section{Author details \\ ${ }^{1}$ Department of Physics and Astronomy, Institute of Applied Physics, and Research Institute of Advanced Materials, Seoul National University, Seoul, Korea. ${ }^{2}$ Department of Material Science and Engineering and Research Institute of Advanced Materials, Seoul National University, Seoul, Korea. ${ }^{3}$ Photonic/Wireless Devices Research Division, Electronics and Telecommunications Research Institute, Daejeon, Korea. ${ }^{4}$ Center for OptoElectronic Materials and Devices, Korea Institute of Science and Technology (KIST), Seoul, Korea}

\section{Conflict of interest}

The authors declare no competing interests.

\section{Publisher's note}

Springer Nature remains neutral with regard to jurisdictional claims in published maps and institutional affiliations.

Supplementary information The online version contains supplementary material available at https://doi.org/10.1038/s41427-021-00301-3.

Received: 12 October 2020 Revised: 17 February 2021 Accepted: 22 February 2021.

Published online: 9 April 2021

\section{References}

1. Chen, R. et al. Nanolasers grown on silicon. Nat. Photonics 5, 170-175 (2011).

2. Yu, G. \& Lieber, C. M. Assembly and integration of semiconductor nanowires for functional nanosystems. Pure Appl. Chem. 82, 2295-2314 (2010).

3. Mazid Munshi, A. \& Weman, H. Advances in semiconductor nanowire growth on graphene. Phys. Status Solidi Rapid Res. Lett. 7, 713-726 (2013).

4. Wang, S., Ren, Z., Guo, Y. \& Gao, P. X. Nano-array integrated monolithic devices: toward rational materials design and multi-functional performance by scalable nanostructures assembly. CrystEngComm 18, 2980-2993 (2016).

5. Sarkar, S. \& Basak, D. Self powered highly enhanced dual wavelength ZnO@CdS core-shell nanorod arrays photodetector: an intelligent pair. ACS Appl. Mater. Interfaces 7, 16322-16329 (2015).

6. Tamboli, A. C. et al. Low-cost CdTe/silicon tandem solar cells. IEEE J. Photovolt. 7, 1767-1772 (2017).

7. Geisz, J. F. et al. Building a six-junction inverted metamorphic concentrator Solar. Cell. IEEE J. Photovolt. 8, 626-632 (2018).

8. Semonin, O. E. et al. Peak external photocurrent quantum efficiency exceeding $100 \%$ via MEG in a quantum dot solar cell. Science $\mathbf{3 3 4}$, 1530-1534 (2011).
9. Adams, J. G. J. et al. Recent results for single-junction and tandem quantum well solar cells. Prog. Photovolt. Res. Appl. 19, 865-877 (2011).

10. Shi, E. et al. Colloidal antireflection coating improves graphene-silicon solar cells. Nano Lett. 13, 1776-1781 (2013).

11. Choi, I. Y. et al. Two-terminal mechanical perovskite/silicon tandem solar cells with transparent conductive adhesives. Nano Energy 65, 104044 (2019).

12. Wang, Y. et al. Stacked dual-wavelength near-infrared organic photodetectors. Adv. Opt. Mater. 28, 1-8 (2020).

13. Vesselinov, M. I. Crystal Growth for Beginners: Fundamentals of Nucleation, Crystal Growth and Epitaxy (World Scientific, 2016).

14. Ayers, J. E., Kujofsa, T., Rago, P. \& Raphael, J. Heteroepitaxy of Semiconductors: Theory, Growth, and Characterization. (CRC Press, 2016).

15. Lee, K. H. et al. Integration of GaAs, GaN, and Si-CMOS on a common 200mm Si substrate through multilayer transfer process. Appl. Phys. Express 9, 086501 (2016).

16. Tanabe, K, Watanabe, K. \& Arakawa, Y. III-V/Si hybrid photonic devices by direct fusion bonding. Sci. Rep. 2, 1-6 (2012).

17. Jain, N. \& Hudait, M. K. III-V Multijunction solar cell integration with silicon: present status, challenges and future outlook. Energy Harvest. Syst. 1, 121-145 (2014).

18. Nakamura, S. GaN growth using GaN buffer layer. Jpn J. Appl. Phys. 30, L1705 (1991).

19. Kim, Y. et al. Remote epitaxy through graphene enables two-dimensional material-based layer transfer. Nature 544, 340-343 (2017).

20. Reina, A. et al. Large area, few-layer graphene films on arbitrary substrates by chemical vapor deposition. Nano Lett. 9, 30-35 (2008).

21. Kim, Y.J., Lee, J.H. \& Yi, G.C. Vertically aligned ZnO nanostructures grown on graphene layers. Appl. Phys. Lett. 95, 213101 (2009).

22. Kim, Y. et al. Position-and morphology-controlled $\mathrm{ZnO}$ nanostructures grown on graphene layers. Adv. Mater. 24, 5565-5569 (2012).

23. Gačević, Ž., Gómez Sánchez, D. \& Calleja, E. Formation mechanisms of gan nanowires grown by selective area growth homoepitaxy. Nano Lett. $\mathbf{1 5}$ 1117-1121 (2015)

24. Chung, K. et al. Growth and characterizations of GaN micro-rods on graphene films for flexible light emitting diodes. APL Mater. 2, 092512 (2014).

25. Hong, Y. J. et al. Van der Waals epitaxial double heterostructure: InAs/singlelayer graphene/InAs. Adv. Mater. 25, 6847-6853 (2013).

26. Koblmüller, G. \& Abstreiter, G. Growth and properties of InGaAs nanowires on silicon. Phys. Status Solidi Rapid Res. Lett. 8, 11-30 (2014).

27. Park, W. I., Yi, G.-C., Kim, J.-W. \& Park, S.-M. Schottky nanocontacts on ZnO nanorod arrays. Appl. Phys. Lett. 82, 4358-4360 (2003).

28. Feng, B. et al. Schottky barrier heights at the interfaces between pure-phase InAs nanowires and metal contacts. J. Appl. Phys. 119, 054304 (2016).

29. Miao, J. et al. High-responsivity graphene/InAs nanowire heterojunction nearinfrared photodetectors with distinct photocurrent on/off ratios. Small 11, 936-942 (2015).

30. Wook Shin, H. et al. Short-wavelength infrared photodetector on Si employing strain-induced growth of very tall InAs nanowire arrays. Sci. Rep. 5, 1-8 (2015).

31. Kind, H., Yan, H., Messer, B., Law, M. \& Yang, P. Nanowire ultraviolet photodetectors and optical switches. Adv. Mater. 14, 158 (2002).

32. Muth, J. F., Kolbas, R. M., Sharma, A. K., Oktyabrsky, S. \& Narayan, J. Excitonic structure and absorption coefficient measurements of $\mathrm{ZnO}$ single crystal epitaxial films deposited by pulsed laser deposition. J. Appl. Phys. 85, 7884-7887 (1999).

33. Adhikari, S. et al. Determining the Fermi level by absorption quenching of monolayer graphene by charge transfer doping. Nanoscale 8, 18710-18717 (2016).

34. Tchoe, Y., Jo, J., Kim, M. \& Yi, G. -C. Catalyst-free growth of $\operatorname{InAs} / \operatorname{In}_{x} G a_{1-x} A s$ coaxial nanorod heterostructures on graphene layers using molecular beam epitaxy. NPG Asia Mater 7, e206-e206 (2015).

35. Patoka, P. \& Giersig, M. Self-assembly of latex particles for the creation of nanostructures with tunable plasmonic properties. J. Mater. Chem. 21, 16783-16796 (2011)

36. Behera, G. \& Ramakrishna, S. A. Enhanced broadband transmission through structured plasmonic thin films for transparent electrodes. J. Nanophotonics $\mathbf{8}$, 083889 (2014).

37. Bae, S. et al. Roll-to-roll production of 30-inch graphene films for transparent electrodes. Nat. Nanotechnol. 5, 574-578 (2010). 